\title{
Characterization of Bone-Marrow-Derived Stem Cells in Osteoporotic Models of the Rat
}

\author{
Julia Goergen, ${ }^{1}$ Sabine Wenisch, ${ }^{2}$ Oksana Raabe, ${ }^{1}$ Andreas Moritz, ${ }^{3}$ Gudrun Schlewitz, ${ }^{4}$ \\ Reinhard Schnettler, ${ }^{4}$ Ute Hempel, ${ }^{5}$ Christian Heiss, ${ }^{4}$ and Stefan Arnhold ${ }^{1}$ \\ ${ }^{1}$ Institute of Veterinary-Anatomy, -Histology and-Embryology of the Justus-Liebig-University Giessen, Frankfurter Straße 98, \\ 35392 Giessen, Germany \\ ${ }^{2}$ Clinic for Small Animals of the Justus-Liebig-University Giessen, Clinical Anatomy and Experimental Surgery, \\ c/o Institute of Veterinary-Anatomy, -Histology and-Embryology, Frankfurter Straße 98, 35392 Giessen, Germany \\ ${ }^{3}$ Department of Veterinary Clinical Sciences of the Justus-Liebig-University Giessen, Pathophysiology and Clinical Pathology, \\ c/o Clinic for Small Animals, Frankfurter Straße 126, 35392 Giessen, Germany \\ ${ }^{4}$ University Hospital of Giessen-Marburg, Department of Trauma Surgery, Rudolf-Buchheim-Straße 7, 35385 Giessen, Germany \\ ${ }^{5}$ Medical Faculty of the Technical University Dresden, Institute of Physiological Chemistry, Fiedlerstraße 42, 01307 Dresden, Germany
}

Correspondence should be addressed to Stefan Arnhold; stefan.arnhold@vetmed.uni-giessen.de

Received 24 May 2013; Accepted 17 June 2013

Academic Editors: A. Chapel, F. Fagioli, and S. M. Hwang

Copyright (C) 2013 Julia Goergen et al. This is an open access article distributed under the Creative Commons Attribution License, which permits unrestricted use, distribution, and reproduction in any medium, provided the original work is properly cited.

\begin{abstract}
Osteoporotic effects observed after osteoporosis induction in the rat by combining ovariectomy (OVX) either with a defined calcium-deficient diet (OVX + Diet) or by administration of a glucocorticoid (dexamethasone) (OVX + Steroid) mimic the skeletal effects observed in humans affected by osteoporosis. In the present investigation rat MSCs have been characterized in vitro after osteoporosis has been induced for twelve weeks in rats by means of OVX $+\operatorname{Diet}(n=5)$ and OVX $+\operatorname{Steroid}(n=5)$. Sham-operated animals $(n=5)$ served as controls. MSCs were harvested from humerus and iliac crest and were cultured in standard medium and in osteogenic differentiation medium for studying the proliferation, migration, and differentiation capacity of the cells. Expression of CD90, CD105, runx2, osteocalcin (OC), and bone sialoprotein (BSP) was performed by using qrtPCR. Calcium deposits developed in the course of osteogenic differentiation were measured by using Pentra 400 Axon Lab. Taken together, the present results showed that osteoporosis induction leads to MSC in a state of senescence: proliferation and migration rates of the cells were diminished pointing to self-renewal deficiency and impaired motility of rat MSC in contrast to controls. However, the osteogenic differentiation capacity was increased after osteoporosis induction with OVX + Diet and OVX + Steroid.
\end{abstract}

\section{Introduction}

By definition of the Consensus Development Conference osteoporosis is a disease characterized by low bone mass and microarchitectural deterioration of bone tissue leading to enhanced bone fragility and a consequent increase in fracture risk (European foundation for osteoporosis 1991). As this disease is restricted to humans [1], there is a great need for animal models in order to validate new therapeutic approaches such as drugs or prosthetic devices.

The most commonly used animal model for osteoporosis is so far the ovariectomized rat [1-3]. In a previous study we have shown that enhanced osteoporotic effects ( $t$ scores below
$-2,5$, reduced bone mineral density and bone mineral content measured by means of Dual Energy X-ray Absorptiometry, DEXA) could be seen in rats by combining ovariectomy with calcium, phosphorus, vitamin c, and vitamin D2/D3 deficiency over a period of 12 weeks [4]. However, little information is available about mesenchymal stem cells (MSCs) residing in the bone marrow of the "osteoporotic" rats.

MSCs are multipotential precursor cells that can differentiate into various cell types such as osteoblasts, chondrocytes, and adipocytes [5]. Due to this differentiation potential combined with their nonimmunogenic characteristics, MSCs are promising therapeutic tools in regenerative medicine 
and tissue engineering [6-9] and thus also for therapeutic strategies in osteoporotic patients.

Despite the frequent use of the rat as the appropriate animal model in osteoporosis research, little is known about osteoporosis-related changes of MSC in the rat. In this study, we therefore focused on characterization of rat MSC three months after osteoporosis had been induced experimentally [4]. Investigations in vitro have been done in order to evaluate putative cellular changes of rat MSC regarding their proliferation, migration, and differentiation capacity. Moreover, investigations were performed in order to evaluate if cellular changes actually correspond to cellular alterations described for MSC of osteoporotic patients [10-12]. Induction of osteoporosis in rats divided into two experimental groups was performed by ovariectomy in combination with a defined calcium-deficient diet and by ovariectomy in combination with steroid. A Sham-operated group was established as a control.

\section{Materials and Methods}

2.1. Experimental Animals. The study protocol was approved by an independent institutional review board prior to surgery and was performed as described previously [4]. In brief, female Sprague Dawley rats $(n=15)$ were divided into two experimental groups and were ovariectomized at an age of 8 weeks. Animals of group 1 (OVX + Diet $)(n=5)$ were fed for 12 weeks with a calcium-deficient diet (calcium, phosphorus, vitamin $c$, and vitamin D2/D3 deficiency). In animals of group 2 (OVX + Steroid) $(n=5)$ osteoporosis induction was performed by the administration of a glucocorticoid (dexamethasone). A sham-operated group (Sham) was established as an internal control $(n=5)$ (group 3). Twelve weeks after the surgery the animals were sacrificed by $\mathrm{CO}_{2}$ asphyxiation. Bone marrow for the isolation of MSC was taken from humerus and iliac crest. These bones were explanted and stored in cold phosphate buffered saline (PBS; Gibco life technologies, Germany) with heparin $(5 \mathrm{U} / \mathrm{mL})$ (Braun, Germany) and 1\% penicillin/streptomycin (P/S; AppliChem, Germany) for the transport to the cell culture lab.

\subsection{Isolation of Mesenchymal Stem Cells and Primary Culture.} The adherent soft tissue was thoroughly debrided of the bones. Then epiphyses were cut off, and the bone marrow was flushed out of the bone with alpha minimum essential medium (alpha MEM; Gibco life technologies, Germany) and a 21 gauche needle (Braun, Germany). The resulting cell suspension was filtered through a $70 \mu \mathrm{m}$ filter (BD Falcon, Belgium), then centrifuged at $250 \mathrm{~g}$ for 5 minutes and washed twice with PBS buffer. Subsequently, the cell pellet was resuspended in $500 \mu \mathrm{L}$ Red Cell Lysis Bbuffer (Sigma, Germany), incubated seven minutes at room temperature, and washed twice with PBS buffer with two centrifugation steps at $250 \mathrm{~g}$ for five minutes. Finally, the cell pellet was resuspended in alpha MEM containing 20\% fetal bovine serum (FBS Gold, PAA, Austria) and $1 \%$ penicillin/streptomycin (P/S; AppliChem, Germany), and the cells were seeded in 6-well culture dishes (Greiner bio-one, Germany) and were incubated at $37^{\circ} \mathrm{C}$ and $5 \% \mathrm{CO}_{2}$.

Culture medium was changed every three days, after washing with PBS prior to the change. When the cells achieved $80 \%$ confluence, they were subcultured. They were lifted from the dishes by treatment with $1 \mathrm{~mL}$ accutase (PAA, Germany) for 5 minutes at $37^{\circ} \mathrm{C}$. Nucleated cell counts were determined with a hemacytometer and seeded at a density of $1 \times 10^{6}$ per $75 \mathrm{~cm}^{2}$ in a culture flask (Greiner bio-one, Germany).

When the cells achieved confluence, they were released from the flasks with accutase, resuspended in freezing medium (alpha MEM, 10\% FBS Gold, 7\% dimethyl sulfoxide (DMSO; AppliChem, Germany)), and stored in liquid nitrogen. For the experiments the cells were thawed and subcultured. In all experiments the cells were used in the third passage.

2.3. Osteogenic Differentiation. For osteogenic differentiation cells were seeded in 6-well and 24-well culture dishes (Greiner bio-one, Germany) at a density of $1 \times 10^{5}$ per $\mathrm{cm}^{2}$. When the cells achieved $80 \%$ confluence, osteogenic differentiation was induced with Dulbecco's Modified Eagle Medium low glucose (DMEM; Gibco life technologies, Germany) containing $10 \%$ FBS Gold, $2.2 \mathrm{mM} \mathrm{CaCl}_{2}$ (SigmaAldrich, Germany), $100 \mathrm{nM}$ dexamethason (Sigma-Aldrich, Germany), $0.3 \mathrm{mM}$ ascorbic acid (Sigma-Aldrich, Germany), $10 \mathrm{mM} \beta$-glycerol phosphate (Sigma-Aldrich, Germany), and $1 \% \mathrm{P} / \mathrm{S}$. The final concentration of $\mathrm{CaCl}_{2}$ amounts to $4 \mathrm{mM}$ since DMEM itself has a $\mathrm{CaCl}_{2}$ concentration of $1.8 \mathrm{mM}$.

This composition of the osteogenic differentiation medium was determined best for the rat bone-marrowderived mesenchymal stem cells in preliminary tests.

Osteogenic stimulation was carried out over 2 weeks. Then the cells were stained for matrix mineralization by using the von Kossa stain. The cells were fixed with $4 \%$ formalin in aqua bidest, washed carefully with distilled water, and then stained with $5 \%$ silver nitrate solution for 30 minutes. Then they were washed again twice and treated with natrium carbonate formaldehyde solution for 5 minutes. After washing with water the cells were treated with Farmers Reducer, consisting of $20 \mathrm{~mL} \mathrm{10 \%}$ natrium thiosulfate and $1 \mathrm{~mL} 10 \%$ formalin. Cell nuclei were stained by nuclear fast red (Merck, Germany). After staining, the cells were embedded in Kaiser's glycerol gelatine (Merck, Germany).

In addition, the cells were harvested for RNA by using peqGOLD TriFast Reagent (Peqlab, Germany).

Calcium deposits in the 24-well culture plates (Greiner bio-one, Germany) were measured by Pentra 400 Axon Lab (ABX Diagnostics, France) in the central laboratory of the clinic for small animals (ECVCP Training Laboratory by the ECVCP Committee for Laboratory Standards) of the JustusLiebig-University in Giessen after dissolving the cultures in $7 \%$ acetic acid. We always measured double cultures. For error correction, we established two different negative controls, namely, DMEM containing 10\% FBS Gold and 1\% $\mathrm{P} / \mathrm{S}$ with $(\mathrm{NCl})$ or without $(\mathrm{NC} 2) 2.2 \mathrm{mM} \mathrm{CaCl}_{2}$. The ultimate 
$\mathrm{CaCl}_{2}$ content was calculated by subtraction of the $\mathrm{CaCl}_{2}$ content of $\mathrm{NCl}$ from the $\mathrm{CaCl}_{2}$ content of the osteogenic differentiated cultures.

2.4. Adipogenic Differentiation. For adipogenic differentiation cells were seeded at a density of $1 \times 10^{5}$ cells $/ \mathrm{cm}^{2}$ in 24-well culture dishes. Adipogenic differentiation was induced when the cells achieved $80 \%$ confluence with DMEM low glucose containing 10\% FBS, $1 \% \mathrm{P} / \mathrm{S}, 1 \mu \mathrm{M}$ dexamethason, $5 \mu \mathrm{g} / \mathrm{mL}$ ITS (Sigma-Aldrich, Germany), $0.2 \mathrm{mM}$ indomethacin (Sigma-Aldrich, Germany), and $0.5 \mathrm{mM}$ IBMX (Sigma-Aldrich, Germany) and conducted over 2 weeks. Then the cells were stained by Oil Red O staining to visualize the fat vacuoles. In brief, the cells were fixed with $4 \%$ PFA, washed three times with aqua bidest, and after that incubated with a newly filtered Oil Red O staining solution for 15 minutes protected from light. After washing with aqua bidest the cells were counterstained with hematoxylin for 15 seconds, then washed with tap water and aqua bidest. After staining, the cells were embedded in Kaiser's glycerol gelatine.

2.5. MTT Assay. To study the proliferation capacity of the cells the MTT assay was carried out. Cells were seeded at a density of $15 \times 10^{3}$ cells $/ \mathrm{cm}^{2}$ in 24 -well culture dishes.

Immediately after seeding, 24 hours and 48 hours after seeding, the medium was replaced by medium containing $0.5 \mathrm{mg} / \mathrm{mL}$ MTT reagent (Sigma, Germany), and then the cells were incubated again for 4 hours. After removal of the MTT medium, the cells were incubated with $200 \mu \mathrm{L}$ DMSO for 10 minutes to dissolve the reduced formazan crystals. The relative number of cells was determined by measuring the optical density of blue formazan crystals, resulting of the reduction of MTT dye in live cells, at $570 \mathrm{~nm}$ with TECAN Sunrise absorbance reader. For each time point we measured triplicate cultures.

To compensate possible variations of the initial cell number and for statistical analysis, the proliferation factor was calculated according to this formula:

$$
\text { proliferation factor }(\mathrm{MTT})=\frac{y(48 \mathrm{~h})}{y(0 \mathrm{~h})} \text {. }
$$

2.6. Migration Assay. In order to study the migration capacity of the cells we used the ibidi culture insert (ibidi, Germany). The cells were seeded at a density of $35 \times 10^{3}$ cells in each well of the insert. After 24 hours the cells were appropriately attached, and the culture insert was carefully removed from the culture dish with sterile tweezers. The consequent cellfree gap is about $500 \mu \mathrm{m}$ wide.

The assay was carried out using the Axio Observer.Z1 microscope based life-cell imaging system by Zeiss (Germany) at $37^{\circ} \mathrm{C}$ and $5 \% \mathrm{CO}_{2}$. Pictures were taken every 10 minutes over at least 36 hours.

The microphotographs were analyzed using the Adobe Photoshop cs5 software, looking at the cell uncovered area in $\%$ over the same time period.
2.7. RNA Extraction and RT-PCR. RNA was extracted from cultured cells using peqGOLD TriFast Reagent and isolated according to the manufacturer's protocol (peqlab, Germany). cDNA was synthesized of mRNA with a concentration of $200 \mathrm{ng} / \mu \mathrm{L}$, after DNA digestion with DNAseI (Roche, Germany), with Gene Amp Gold Core Kit recording to the manufacturer's protocols (Invitrogen, Germany).

2.8. Quantitative Real-Time PCR. Quantitative real-time PCR (qRT-PCR) was performed with QuantiFast SYBR Green PCR Kit recording to the manufacturer's protocol (Quiagen, Germany). All primers were Quantitect Primers purchased from Quiagen for bone sialoprotein (BSP) (Cat. no: QT02333296), osteocalcin (OC) (Cat. no: QT01084573), Runt-related transcription factor 2 (runx2) (Cat. no: QT01620647), endoglin (CD105) (Cat. no: QT01689989), cluster of differentiation 90 (CD90) (Cat. no: QT00195825), and a housekeeping gene, glyceraldehyde 3-phophate dehydrogenase (GAPDH) (Cat. no: QT00199633). The primers were designed and tested by the manufacturer. The PCR reactions were carried out in triplicates.

qRT-PCR was carried out with the following protocol: 5 minutes $95^{\circ} \mathrm{C}, 10$ seconds $95^{\circ} \mathrm{C}$, and 30 seconds $60^{\circ} \mathrm{C}$; 39 cycles. Melt curve: $60^{\circ} \mathrm{C}$ to $95^{\circ} \mathrm{C}$, increment $0.5^{\circ} \mathrm{C}$ for 5 seconds. Data was analyzed using the CFX manager software 2.0 (Bio-Rad, Germany).

2.9. Statistics. Statistical analysis was performed using a two and one factor analysis of variance. $P \leq 0.05$ was taken as significant.

\section{Results}

3.1. Cell Culture. Cultivating the cells in the primary culture we first observed that the cells of the ovariectomized rats (group 1: OVX + Diet, group 2: OVX + Steroid) tended to have slower growth characteristics and had a slightly different morphology compared to the cells of the control group (group 3: Sham). They were more broad and flattened, whereas the cultures of the control group were composed of cells with spindle-shaped morphology (Figure 1). The cells of all groups (group 1-3) were adherent to plastic in standard culture conditions. Stem cell characteristics were further analyzed and confirmed by the usual tests like the expression of specific surface markers and by the analysis of pluripotency.

\subsection{Osteogenic Differentiation}

Matrix Mineralization: Von Kossa Staining and Calcium Measurement. Looking at the differentiation capacity of the cells of all experimental groups, the cells of ovariectomized rats (group 1: OVX + Diet and group 2: OVX + Steroid) showed a higher matrix mineralization in contrast to control cells. We examined matrix mineralization by means of von Kossa staining and calcium measurement (Figures 2 and 3). The levels of calcium measured in the negative controls ( $\mathrm{NC1}$ mean: $0,113 \mathrm{mmol} / \mathrm{L}, \mathrm{NC} 2$ mean: $0 \mathrm{mmol} / \mathrm{L})$ were next to zero, and the differences between osteogenic differentiated 


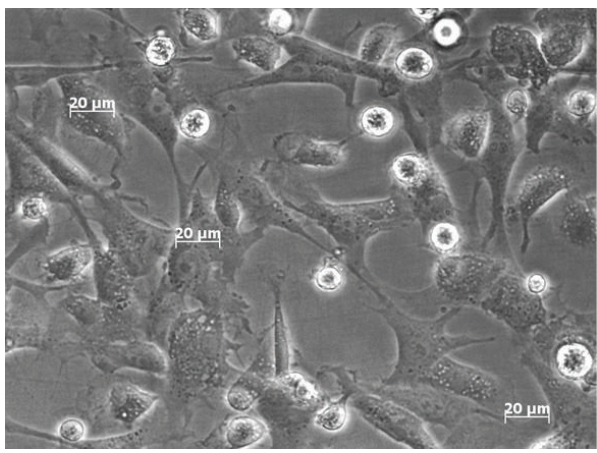

(a)

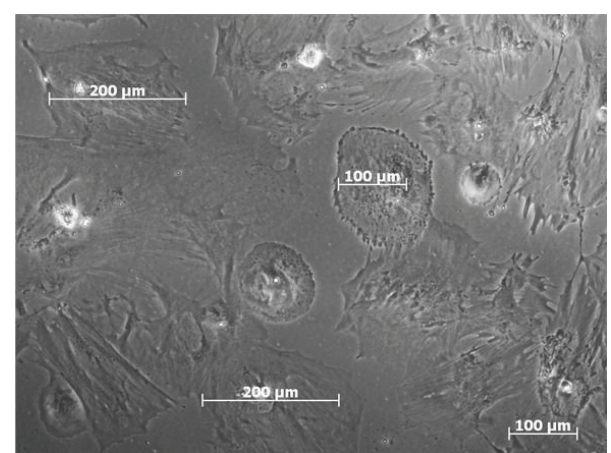

(b)

FIGURE 1: Cell morphology. The microphotographs are representative of the different cell morphologies of MSC taken from the Sham group (a) and of ovariectomized rats as shown here for the OVX + Diet group (b). The MSCs of the Sham group (a) are smaller and have a rather spindle-shaped cell morphology, whereas cells of the OVX + Diet group (b) mainly consisted of a more broad and flattened cell type.

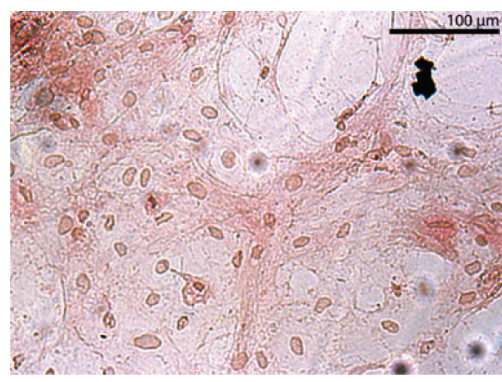

(a)

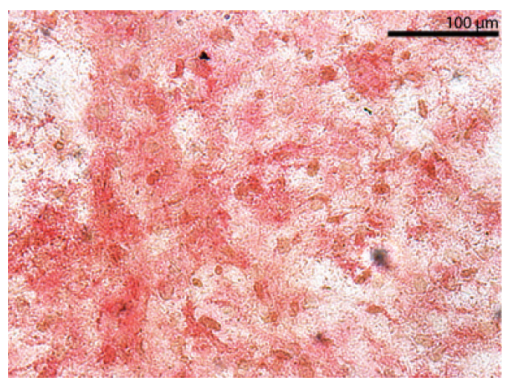

(d)

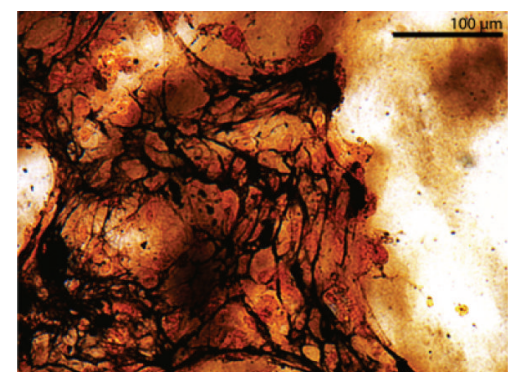

(b)

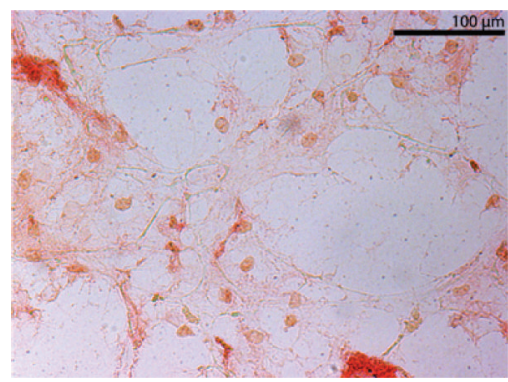

(e)

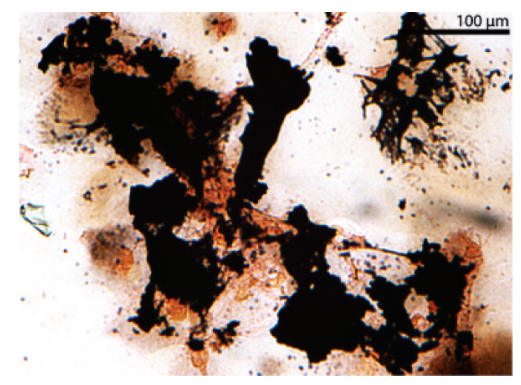

(c)

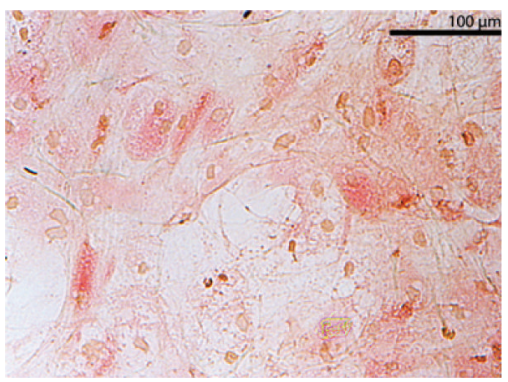

(f)

Figure 2: Osteogenic differentiation: von Kossa staining. Von Kossa staining revealed that cells of ovariectomized rats ((b) OVX + Diet, (c) OVX + Steroid) showed more matrix mineralization compared to the control group ((a) Sham), whereas no staining for mineralized matrix could be seen in cells treated with the control medium (NC2) ((d) Sham, (e) OVX + Diet, and (f) OVX + Steroid).

cells and the corresponding negative controls were highly significant $(P \leq 0,0001)$.

3.3. Adipogenic Differentiation. Cells of animals of group 1 (OVX + Diet) and of group 3 (Sham) showed marked adipogenic differentiation capacity as was demonstrated by numerous lipid vacuoles within the cytoplasm visualized by the Oil Red O staining. Cells of OVX + Steroid animals had a rather weak adipogenic differentiation potential due to the small amount of lipid vacuoles (Figure 4).

3.4. Quantitative Real-Time PCR. The cells of animals of all the three groups expressed the stem cell markers CD105 and CD90 (Data not shown) [5].
qRT-PCR analyses showed that the mRNA expressions of bone sialoprotein (BSP), osteocalcin (OC), and Runt-related transcription factor 2 (runx2) were upregulated due to the exposure of the cells to the osteogenic induction medium compared to the negative control (NC2) (Figure 5). These differences between the mRNA expression of osteogenic differentiated cells and of the negative controls were highly significant $(P \leq 0,0001)$. Highest mRNA expression of BSP, OC and runx 2 could be seen in samples of OVX + Diet (group 1) animals. The lowest expression of BSP and OC is shown by OVX + Steroid, while cells of the Sham-operated animals (group 3) showed the lowest expression of runx2 (Figure 5).

The mRNA expression of runx 2 of undifferentiated cells, respectively, in the negative controls was the highest in cells 


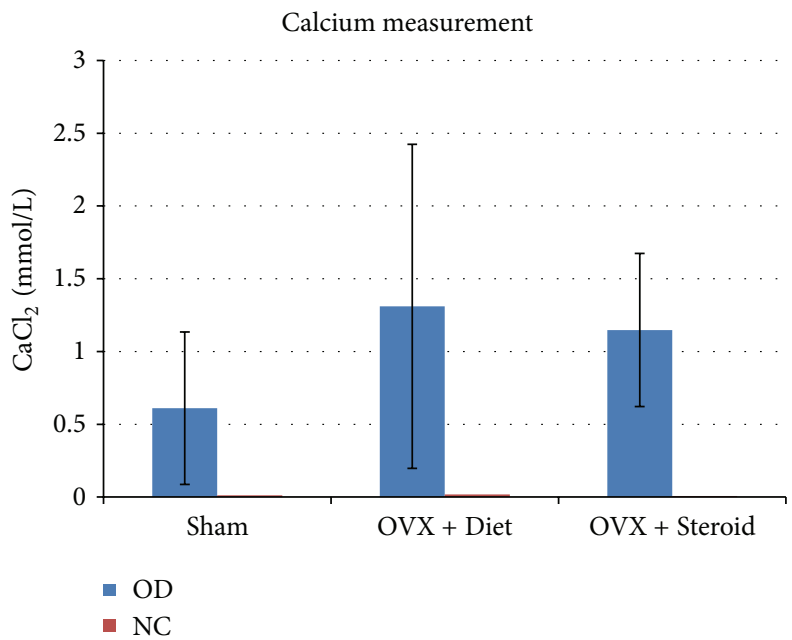

FIGURE 3: Calcium measurement of osteogenic differentiation. Calcium measurement of calcium deposits of the cultures matches the results of the von Kossa staining. Cells of OVX + Diet showed the highest $\mathrm{CaCl}_{2}$ levels, followed by OVX + Steroid and Sham. Cells cultivated in the control medium showed $\mathrm{CaCl}_{2}$ levels next to zero. OD: osteogenic differentiated cells; $\mathrm{NC}$ : negative control.

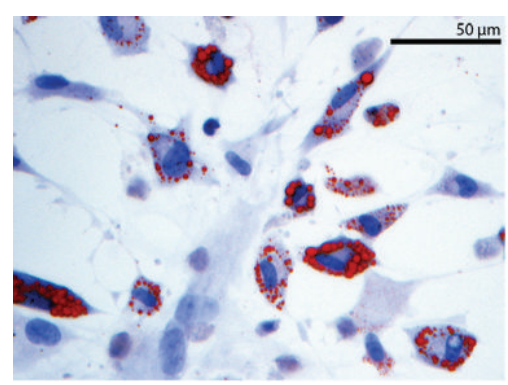

(a)

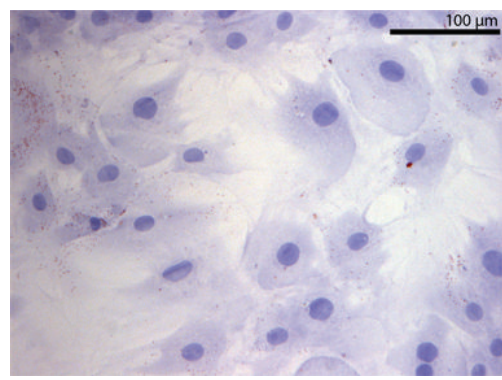

(d)

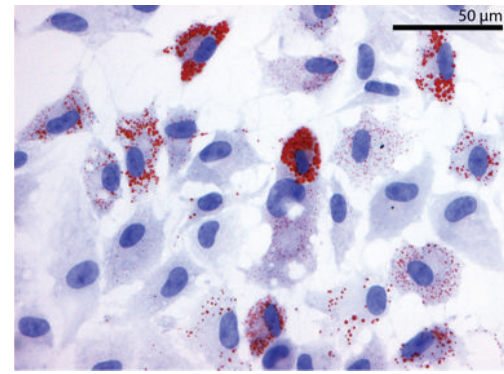

(b)

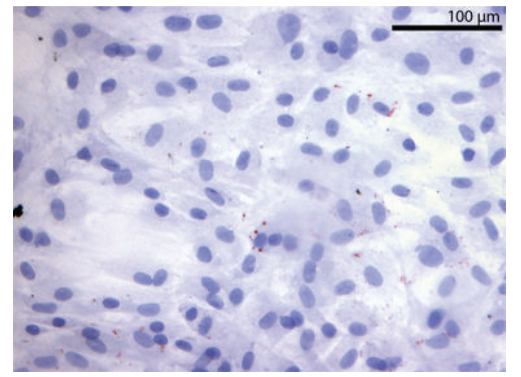

(e)

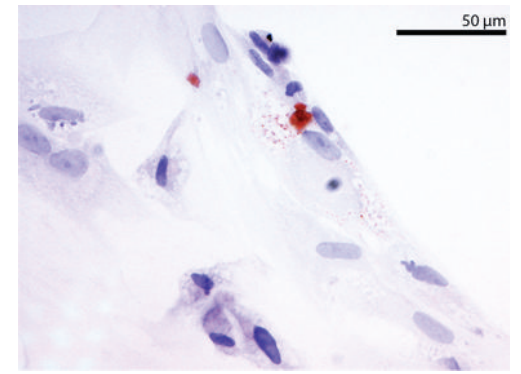

(c)

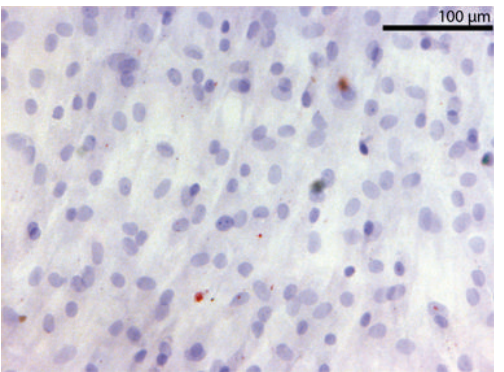

(f)

FIgURE 4: Adipogenic differentiation: Oil Red O staining. Oil Red O staining shows that the cells were able to differentiate towards the adipogenic lineage. Cells of the control group $(\operatorname{Sham}=(\mathrm{a}))$ and cells of animals of the OVX + Diet $(\mathrm{b})$ group showed an obvious adipogenic differentiation. Cells of OVX + Steroid (c) animals had a rather weak adipogenic differentiation potential. Cells cultivated in the control medium showed no fat vacuoles stained by Oil Red O ( d $)=$ Sham, $(e)=$ OVX + Diet, and (f) = OVX + Steroid).

of OVX + Diet (group 1) animals and the lowest in cells of animals of the Sham group (group 3).

The differences between the groups looking at the expression of runx 2 of the undifferentiated cells were significant $(P=0,0058)$.

3.5. Cell Migration. In order to compare the MSCs regarding their ability to migrate we conducted migration assays using cells of five animals from each group. The analysis of the microphotographs revealed that cells of ovariectomized rats of both experimental groups (OVX + Diet and OVX + Steroid) need a longer time period to close the cell free gap compared to the control group (Sham). In detail, the cells of animals of group 1 (OVX + Diet) showed the lowest migration capacity. Sometimes the cells were not able to close the celluncovered area in a time frame of 48 hours (Figures 6 and 7).

3.6. Cell Proliferation. The MTT assay showed that until 24 hours the cells of the three different groups had nearly the 


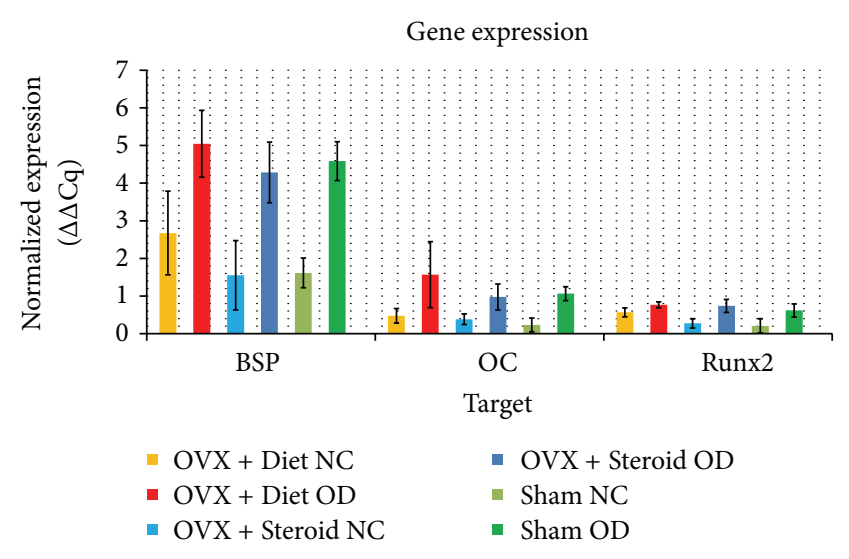

FIGURE 5: Quantitative real-time PCR of osteogenic differentiation markers BSP, OC, and runx2. The figure shows the expression levels of bone sialoprotein (BSP), osteocalcin (OC), and Runt-related transcription factor 2 ( $r u n x 2)$ of cells of all groups (Sham; OVX + Diet; OVX + Steroid), comparing the expression levels of the cells grown in the osteogenic differentiation medium (OD) or in the control medium (NC).

same proliferation capacity, but after 48 hours a lower cell proliferation of the cells of the ovariectomized rats became visible. According to the results of cell migration the cells of group 1 (OVX + Diet) showed the lowest cell proliferation capacity compared to group 3 (Sham) (Figure 8).

\section{Discussion}

This study has characterized mesenchymal stem cells from two rat osteoporosis models. The present investigations have shown that induction of osteoporosis by means of ovariectomy in combination with a calcium-deficient diet or application of a steroid resulted in lower proliferation rates and migration capacity of bone-marrow-derived MSC of the rat, while the osteogenic differentiation potential of the cells increased.

The stem cells within the cultures have been identified due to their capacity to differentiate into the osteogenic and adipogenic lineages, their antigenic profile concerning the expression of the stem cell markers CD105 and CD90 (data not shown), and additionally their plastic adherence [5].

As presently observed cultures of MSCs contain two different types of adherent cells, spindle-formed, rapidly dividing cells, and slower dividing broad and flattened cells [13]. The broad and flattened cell type is described as the phenotype of cells which are in a state of replicative senescence [14] and was mainly observed in cultures derived from ovariectomized rats (OVX + Diet/Steroid), while cells from animals of the sham operated control group were predominantly spindle shaped. Accordingly, young and aged rats also revealed age related changes of morphology and selfrenewal capacity of bone-marrow-derived MSC [15].

The predominance of cells in a state of replicative senescence [14] in cultures derived from ovariectomized rats (OVX + Diet/Steroid) is in line with our observations regarding both, the low proliferation and the low migration capacity of these cells.

These findings might indicate that the induction of osteoporosis in the rat results in accelerated aging of the MSCs of the bone marrow. Aging of an organism might be the result of stem cell aging leading to reduced capacity of regeneration [9]. An impairment of the migration capacity of MSC observed 12 weeks after induction of osteoporosis might be interpreted as a disability of the cells to migrate to the site of bone loss.

The low proliferation capacity of the cells might correlate with a reduced self-renewal capacity and might lead to a gradual depletion of MSC resources in the bone marrow with time. Accordingly, Sca-1 ${ }^{-1-}$ mice develop an agedependent osteoporosis because of mesenchymal progenitor self-renewal deficiency [16]. Moreover, human MSCs obtained from osteoporotic patients [10] reveal lower proliferation rates than control cells.

The statistical analysis of the results of the MTT assay did not reveal significant differences between the groups of rats due to interindividual variations within the experimental groups. Nevertheless dealing with interindividual variations is a common and well-known difficulty working with mesenchymal stem cells $[17,18]$.

Looking at the osteogenic differentiation potential of the MSCs it became obvious that cells of the ovariectomized animals (group 1: OVX + Diet and group 2: OVX + Steroid) had an even greater osteogenic differentiation potential than the cells of group 3 (Sham). Particularly cells of group 1 (OVX + Diet) showed the highest expression levels of osteocalcin, BSP, and runx2. These findings are not in line with the enhanced osteoporotic effects observed in OVX + Diet rats by means of DEXA [4]. Possibly, the upregulation of the osteogenic markers might represent a compensatory mechanism at the cellular level, and the amount of the markers seizes with time.

Looking at the undifferentiated cells, the differences of the runx2 expression were significant $(P=0,0058)$. These results may represent the status of the cells of the OVX + Diet animals in vivo, being in a direction towards the osteogenic lineage to compensate the proceeding bone loss.

The significant differences between the different cell treatments, namely, osteogenic differentiation versus the negative controls, expose the established method of osteogenic differentiation and confirm our results.

Human MSCs derived from bone marrow of osteoporotic donors either maintain [11] or diminish [10] their differentiation capacity. These contradictory results might be a result of donor variability including individual differences concerning the osteoporotic status of the patients/donors. Accordingly, investigations on human MSC isolated from bone marrow of osteoporotic patients have demonstrated marked differences of cell behaviour: while Justesen et al. (2002) described a cellular differentiation capacity of MSC derived from osteoporotic patients which was similar to agematched controls [11], other authors showed that the proliferation capacity and the osteogenic differentiation potential of MSCs obtained from osteoporotic postmenopausal women 


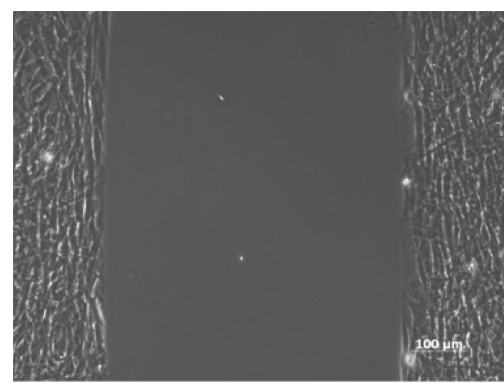

(a)

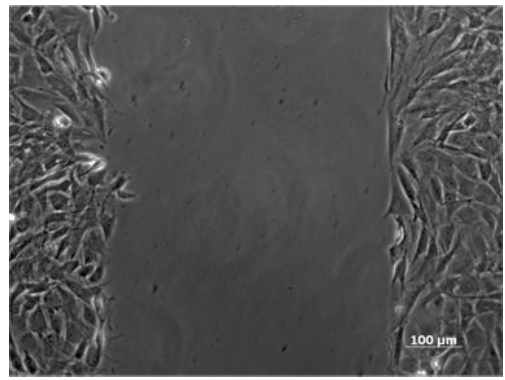

(d)

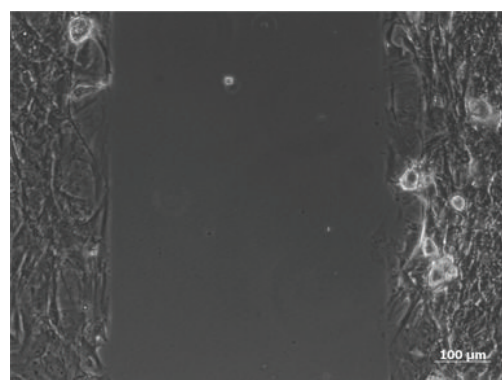

(g)

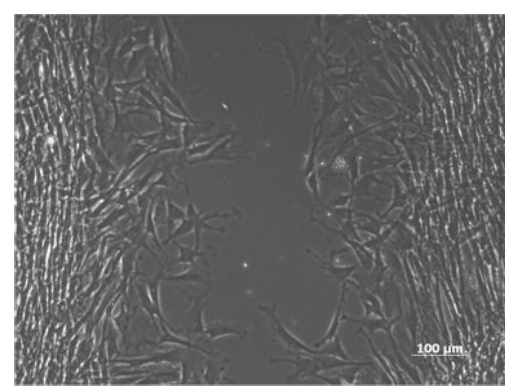

(b)

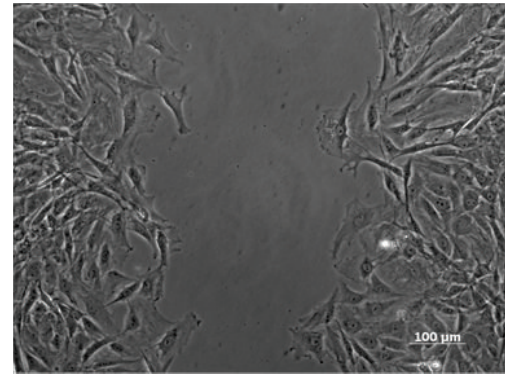

(e)

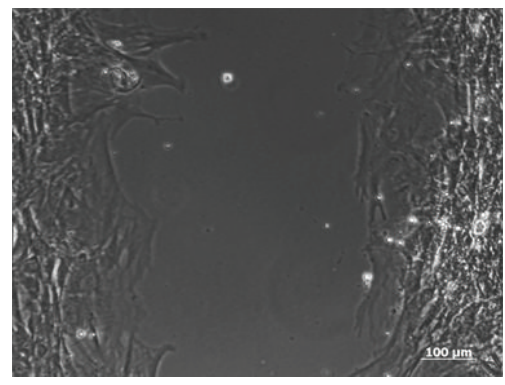

(h)

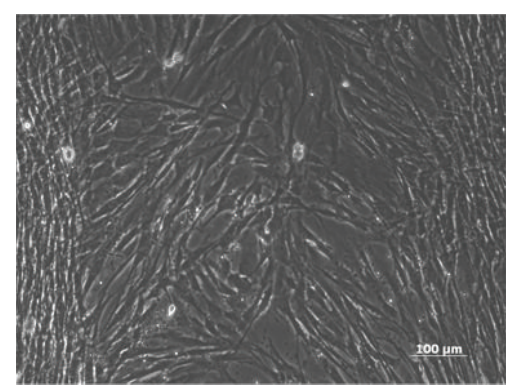

(c)

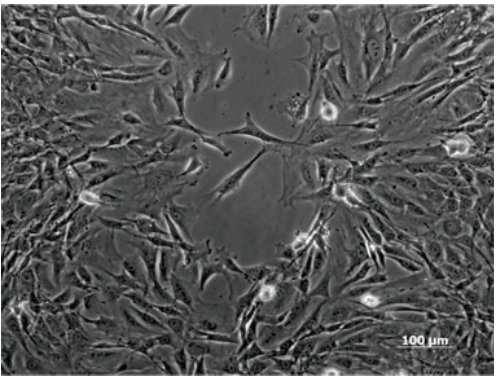

(f)

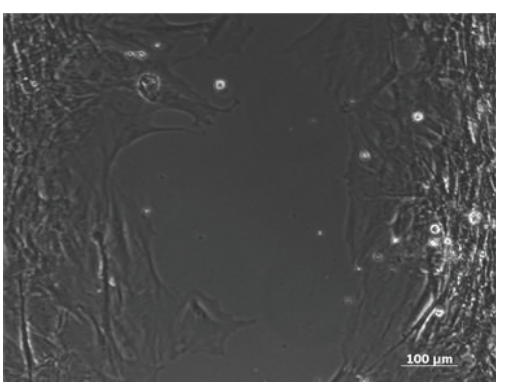

(i)

FIGURE 6: Images of cell migration. Images show the time course of the migration comparing the cells of the different groups. The pictures show the lower migration capacity of the MSC from ovariectomized rats (OVX + Diet (images (g) -(i)) and OVX + Steroid (images (d) - (f))) in contrast to group 3 (Sham (images (a)-(c))). (a) Sham, 0 hours; (b) Sham, 12 hours; (c) Sham, 24 hours; (d) OVX + Steroid, 0 hours; (e) OVX + Steroid, 12 hours; (f) OVX + Steroid, 24 hours; (g) OVX + Diet, 0 hours; (h) OVX + Diet, 12 hours; (i) OVX + Diet, 24 hours.

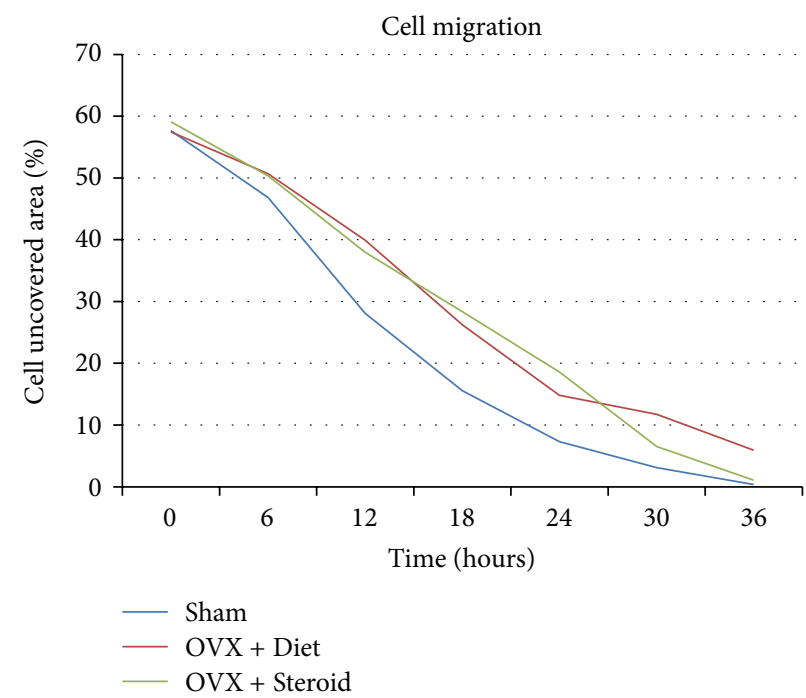

FIGURE 7: Analysis of cell migration. These data show that cells of ovariectomized rats of both groups OVX + Diet and OVX + Steroid need a longer time period to close the cell free gap compared to the Sham group. Note that the cells of the diet group showed the lowest migration capacity. 


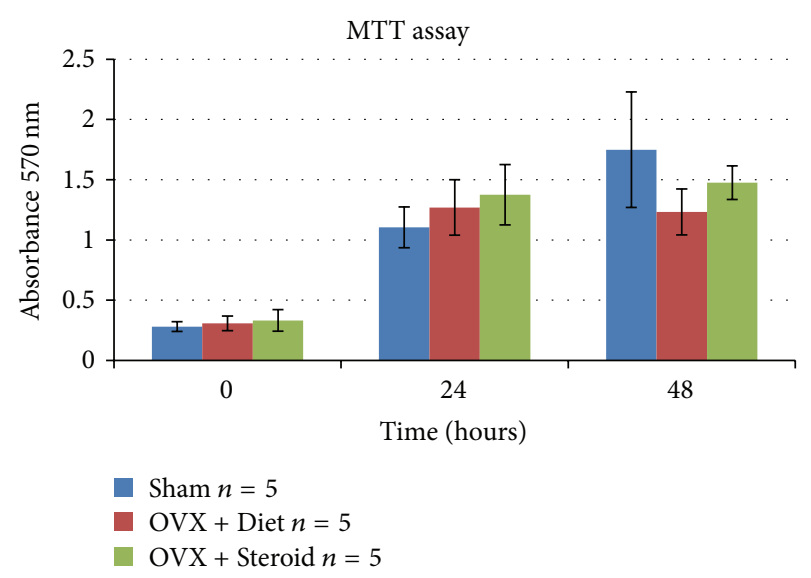

FIGURE 8: Cell proliferation as analyzed by the MTT assay. MTT assay shows that after 48 hours one could see a lower cell proliferation of the cells from the ovariectomized rats (OVX + Diet and OVX + Steroid) compared to group 3 (Sham).

were decreased in contrast to controls [10]. Additionally, it has been observed that cells derived from osteoporotic donors produce an extracellular matrix deficient in type 1 collagen resulting in an inability to sustain proper mineralization [12].

In conclusion the presented data point out a predominance of stem cells derived from the osteoporotic models to be in a state of senescence irrespective of the osteoporosis inducers administered, which became obvious by the decreased proliferation and self-renewing capacity as well as by the impaired migration rates. However, surprisingly the differentiation capacity into the osteogenic lineage was increased in both experimental groups (OVX + Diet and OVX + Steroid).

Based upon the present results it could be shown that investigations on rat MSC derived from realistic osteoporosis models give interesting insights into osteoporosis-related changes of stem cells and provide a suitable model to understand the underlying mechanisms of multifactorial osteoporosis in humans.

\section{Conflict of Interests}

The authors of the paper do not have a direct financial relation with the commercial identities mentioned in the paper, especially not with the Adobe company (Adobe, Munich, Germany, adobe Photoshop cs5 software).

\section{Acknowledgments}

This work was supported by a Grant from the DFG (Collaborative Research Center TR 79). The authors would like to thank the Department of Bioinformatics (Dr. Klaus Failing) of the veterinary faculty of Giessen for the statistical analysis and Annika Goessl for the expert technical assistance.

\section{References}

[1] A. S. Turner, "Animal models of osteoporosis-necessity and limitations," European Cells and Materials, vol. 1, pp. 66-81, 2001.
[2] H. M. Frost and W. S. S. Jee, "On the rat model of human osteopenias and osteoporoses," Bone and Mineral, vol. 18, no. 3, pp. 227-236, 1992.

[3] P. P. Lelovas, T. T. Xanthos, S. E. Thorma, G. P. Lyritis, and I. A. Dontas, "The laboratory rat as an animal model for osteoporosis research," Comparative Medicine, vol. 58, no. 5, pp. 424-430, 2008.

[4] C. Heiss, P. Govindarajan, G. Schlewitz et al., "Induction of osteoporosis with its influence on osteoporotic determinants and their interrelationships in rats by DEXA," Medical Science Monitor, vol. 18, pp. BR199-BR207, 2012.

[5] M. Dominici, K. Le Blanc, I. Mueller et al., "Minimal criteria for defining multipotent mesenchymal stromal cells. The International Society for Cellular Therapy position statement," Cytotherapy, vol. 8, no. 4, pp. 315-317, 2006.

[6] K. Nohroudi, S. Arnhold, T. Berhorn, K. Addicks, M. Hoehn, and U. Himmelreich, "In vivo MRI stem cell tracking requires balancing of detection limit and cell viability," Cell Transplantation, vol. 19, no. 4, pp. 431-441, 2010.

[7] F. Ayaloglu-Butun, E. Terzioglu-Kara, Z. Tokcaer-Keskin, and K. C. Akcali, "The effect of estrogen on bone marrow-derived rat mesenchymal stem cell maintenance: inhibiting apoptosis through the expression of Bcl-xL and Bcl-2," Stem Cell Reviews and Reports, vol. 8, pp. 393-401, 2012.

[8] D. Chanda, S. Kumar, and S. Ponnazhagan, "Therapeutic potential of adult bone marrow-derived mesenchymal stem cells in diseases of the skeleton," Journal of Cellular Biochemistry, vol. 111, no. 2, pp. 249-257, 2010.

[9] A. Wilson, L. A. Shehadeh, H. Yu, and K. A. Webster, "Agerelated molecular genetic changes of murine bone marrow mesenchymal stem cells," BMC Genomics, vol. 11, no. 1, article 229, 2010.

[10] J. P. Rodriguez, S. Garat, H. Gajardo et al., "Abnormal osteogenesis in osteoporotic patients is reflected by altered mesenchymal stem cells dynamics," Journal of Cellular Biochemistry, vol. 75, pp. 414-423, 1999.

[11] J. Justesen, K. Stenderup, E. F. Eriksen, and M. Kassem, "Maintenance of osteoblastic and adipocytic differentiation potential with age and osteoporosis in human marrow stromal cell cultures," Calcified Tissue International, vol. 71, no. 1, pp. 3644, 2002.

[12] J. P. Rodriguez, L. Montecinos, S. Rios et al., "Mesenchymal stem cells from osteoporotic patients produce a type I collagendeficient extracellular matrix favoring adipogenic differentiation," Journal of Cellular Biochemistry, vol. 79, pp. 557-565, 2000.

[13] D. C. Colter, I. Sekiya, and D. J. Prockop, "Identification of a subpopulation of rapidly self-renewing and multipotential adult stem cells in colonies of human marrow stromal cells," Proceedings of the National Academy of Sciences of the United States of America, vol. 98, no. 14, pp. 7841-7845, 2001.

[14] R. Tuli, S. Tuli, S. Nandi et al., "Characterization of multipotential mesenchymal progenitor cells derived from human trabecular bone," Stem Cells, vol. 21, no. 6, pp. 681-693, 2003.

[15] F. Z. Asumda and P. B. Chase, "Age-related changes in rat bonemarrow mesenchymal stem cell plasticity," BMC Cell Biology, vol. 12, article 44, 2011.

[16] M. Bonyadi, S. D. Waldman, D. Liu, J. E. Aubin, M. D. Grynpas, and W. L. Stanford, "Mesenchymal progenitor self-renewal deficiency leads to age-dependent osteoporosis in Sca-1/Ly-6A null mice," Proceedings of the National Academy of Sciences of the United States of America, vol. 100, no. 10, pp. 5840-5845, 2003. 
[17] L. Moroni and P. M. Fornasari, "Human mesenchymal stem cells: a bank perspective on the isolation, characterization and potential of alternative sources for the regeneration of musculoskeletal tissues," Journal of Cellular Physiology, vol. 228, pp. 680-687, 2013.

[18] P. Benisch, T. Schilling, L. Klein-Hitpass et al., "The transcriptional profile of mesenchymal stem cell populations in primary osteoporosis is distinct and shows overexpression of osteogenic inhibitors," PLoS One, vol. 7, article e45142, 2012. 


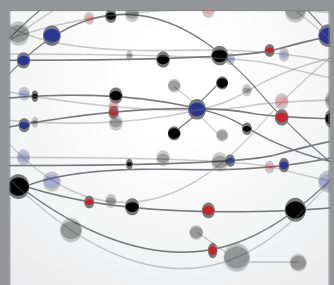

The Scientific World Journal
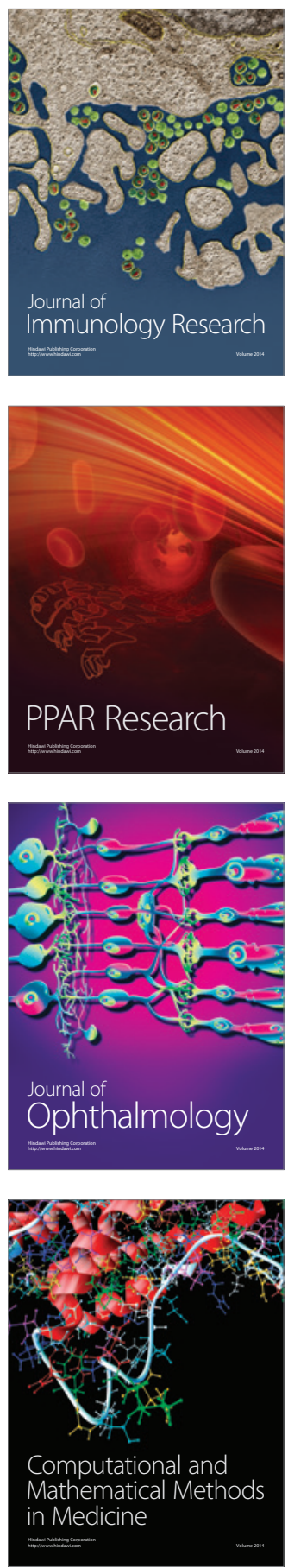

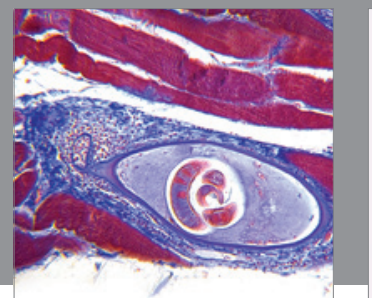

Gastroenterology

Research and Practice
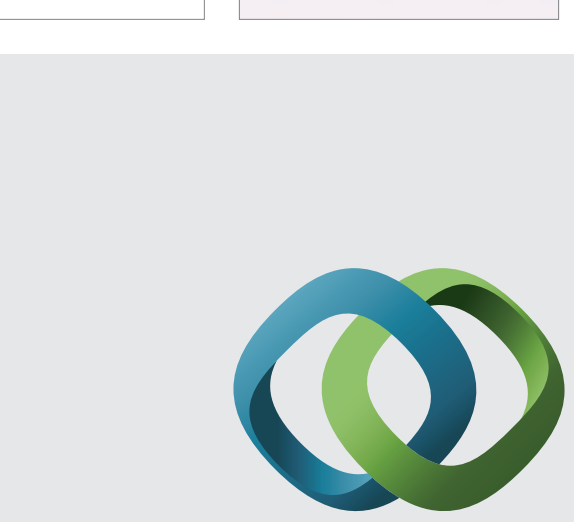

\section{Hindawi}

Submit your manuscripts at

http://www.hindawi.com
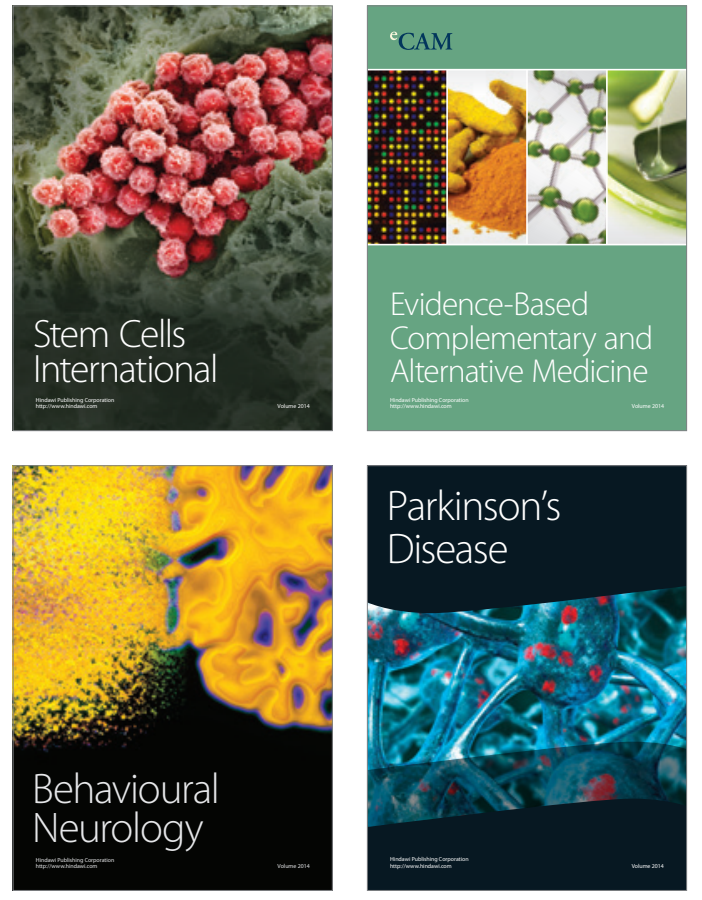
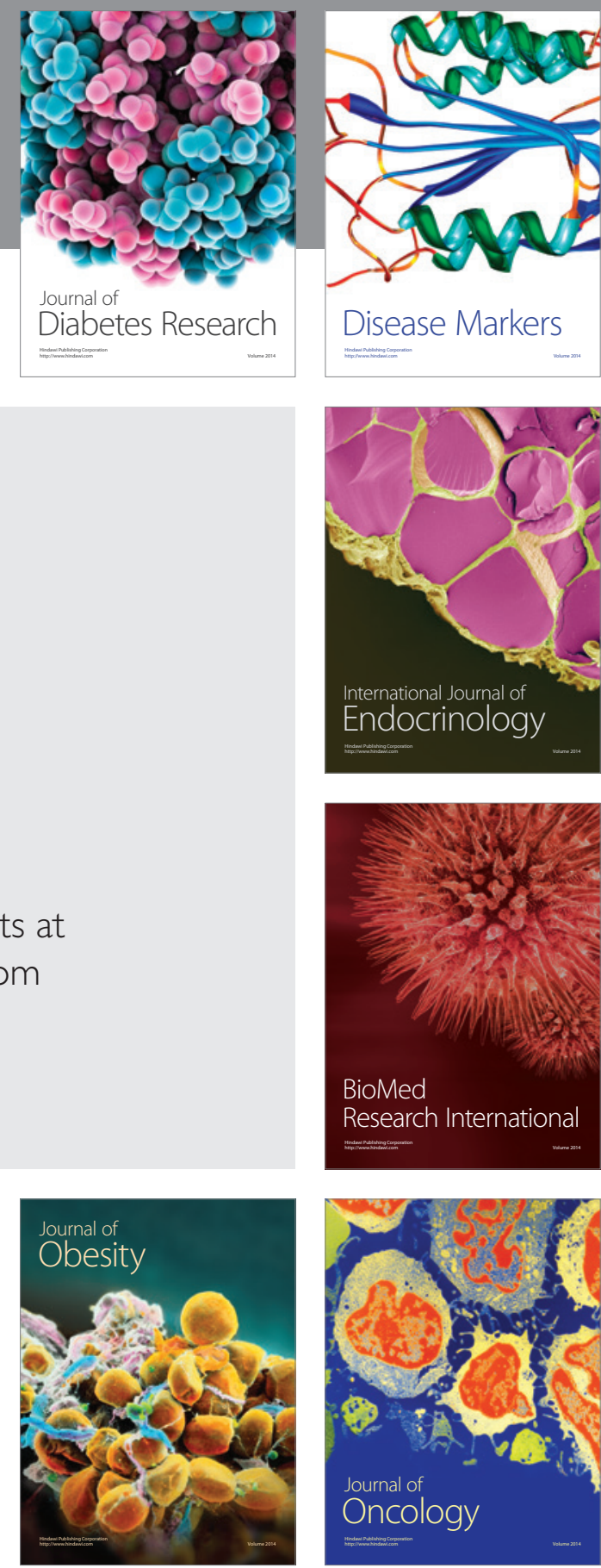

Disease Markers
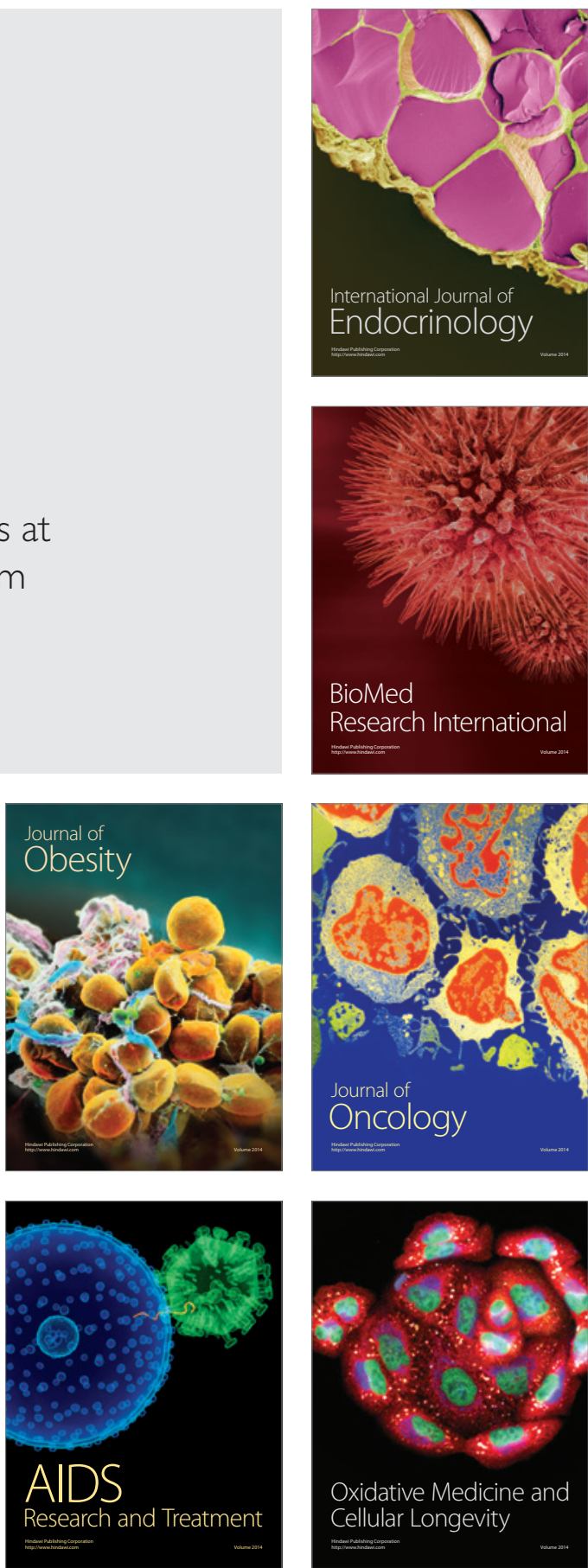